\title{
Sweep maps for lattice paths (extended abstract)
}

\author{
Nicholas A. Loehr $\left.{ }^{1,2}\right|^{k}$ and Gregory S. Warrington $\|^{\prime}$ \\ ${ }^{1}$ Dept. of Mathematics, Virginia Tech, Blacksburg, VA 24061-0123, USA \\ ${ }^{2}$ Mathematics Dept., United States Naval Academy, Annapolis, MD 21402-5002, USA \\ ${ }^{3}$ Dept. of Mathematics and Statistics, University of Vermont, Burlington, VT 05401, USA
}

\begin{abstract}
.
Sweep maps are a family of maps on words that, while simple to define, are not yet known to be injective in general. This family subsumes many of the "zeta maps" that have arisen in the study of $q, t$-Catalan numbers in the course of relating the three statistics of area, bounce and dinv. A sweep map can be defined for words over arbitrary alphabets with arbitrary weights. The latter property makes them particularly suitable for the study of rational Catalan combinatorics.

Résumé.

Applications de balayage sont une famille de applications sur les mots qui, bien que simple à définir, ne sont pas encore connus pour être injective en général. Cette famille englobe bon nombre des «applications zeta» qui sont apparues dans l'étude de les numéros $q, t$-Catalan dans le cadre de relier les trois statistiques de la area, bounce et dinv. Un application de balayage peut être définie pour les mots sur alphabets arbitraires avec des poids arbitraires. Cette derniére propriété rend particulièrement adapté à l'étude de rationnel Catalan combinatoire.
\end{abstract}

Keywords: $q, t$-Catalan numbers, Dyck paths, zeta map, diagonal harmonics, nabla operator

\section{Introduction}

We introduce a family of sorting maps on words that we call sweep maps. In its simplest form, a sweep map $\mathrm{sw}_{r, s}$ uses coprime parameters $r$ and $s$ to associate a level $l_{i}$ to each letter $w_{i}$ in a word $w=$ $w_{1}, \ldots, w_{n}$ over the alphabet $\{N, E\}$. (Note that $r$ or $s$ may be negative.) This assignment is done recursively: Using the convention that $l_{0}=0$, for $i \geq 1$ we set $l_{i}=l_{i-1}+r$ if $w_{i}=N$ and $l_{i}=l_{i-1}+s$ if $w_{i}=E$. The word $\mathrm{sw}_{r, s}(w)$ is then obtained by sorting the letters in $w$ according to level, starting with $-1,-2,-3, \ldots$ then continuing with $\ldots, 2,1,0$. Figure 1 provides an example of $\mathrm{sw}_{5,-3}$ acting on the word $w=E N E E N N E E$. (Here we have identified $w$ with a lattice path in the plane: Each $N$ corresponds to a unit-length north step while each $E$ corresponds to a unit-length east step.)

\footnotetext{
*Email: nloehrevt.edu. This work was partially supported by a grant from the Simons Foundation (\#244398 to Nicholas Loehr).

$\dagger$ Email: gregory . warrington@uvm. edu. Supported in part by National Science Foundation grant DMS-1201312.

1365-8050 @ 2014 Discrete Mathematics and Theoretical Computer Science (DMTCS), Nancy, France
} 


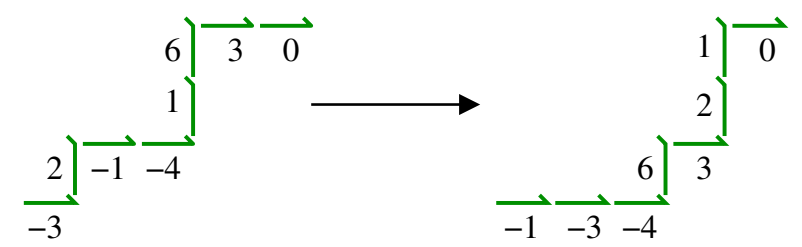

Fig. 1: The action of the sweep map $\mathrm{sw}_{5,-3}$ on the word $w=E N E E N N E E$. Next to each step in $w$ is written its level, $l_{i}$. Each step in $\mathrm{sw}_{5,-3}(w)$ has been labeled by the level of the corresponding step in $w$.

Paradoxically, even though sweep maps act by sorting, they are (apparently) injective. The reader may find it useful to check this injectivity by hand for $\mathrm{sw}_{3,-2}$ acting on the set of all lattice paths from $(0,0)$ to $(3,2)$. As detailed in Conjecture 3.3 , injectivity seems to hold even for the general sweep maps over arbitrary alphabets with arbitrary weights. The injectivity of the general sweep maps appears to be a very subtle and difficult fact. See [3] for a review of the special cases in which an algorithm for the inverse of a particular sweep map is known.

The sweep maps encode complex combinatorial information related to $q, t$-Catalan numbers, to the Bergeron-Garsia nabla operator, and to other constructions arising in representation theory, algebraic geometry, and symmetric functions. Many researchers have discovered special cases of the sweep map in many different guises over the last fifteen years or so. One of the goals of this paper is to present a general unifying framework for all of this work. In [14, 15, 16], the first author introduced bijections on $m$-Dyck paths, as well as generalizations to lattice paths contained in certain trapezoids, that turn out to be special cases of the sweep map. The bijection in the case $m=1$ also appears in a paper of Haglund and Loehr [13] and is foreshadowed by a counting argument in Haglund's seminal paper on $q, t$-Catalan numbers [12, proof of Thm. 3.1]. The inverse bijection in the case $m=1$ appears even earlier, where it was used by Andrews et al. [1] in their study of $a d$-nilpotent $\mathfrak{b}$-ideals in the Lie algebra $\mathfrak{s l}(n)$. (See also [19].) More recently, special cases of the sweep map have arisen while studying lattice paths in squares [18]; partition statistics [17]; simultaneous core partitions [2]; and compactified Jacobians [10, 11]. We discuss a number of these connections in more detail in Section 4

We suspect that to the mathematician-on-the-street, the most interesting question regarding the sweep maps is whether they are injective (as conjectured in Conjecture 3.3. For a researcher interested in the $q, t$-Catalan numbers, however, of comparable interest is the connection between the sweep maps and statistics on lattice paths such as area, bounce and dinv. Since shortly after Haiman's introduction of dinv it has been known that a "zeta map" takes dinv to area to bounce. One point of view, then, is that rather than having three statistics on Dyck paths, we have one statistic — area — and a sweep map. Many polynomials related to the $q, t$-Catalan can be defined using only an "area" and an appropriate sweep map. That these polynomials are jointly symmetric (conjecturally) supports the utility of this view (see Section 5.

The structure of this paper is as follows. Section 2 introduces the necessary background on lattice paths. We then define sweep maps and present Conjecture 3.3 on their injectivity in Section 3 . Section 4 reviews various algorithms that have appeared in the literature that are equivalent to the sweep map. Finally, Section 5 shows how the sweep maps may be used to give concise combinatorial formulas for the higher $q, t$-Catalan numbers and many related polynomials that arise by applying the nabla operator to appropriate symmetric functions and then extracting the coefficient of $s_{\left(1^{n}\right)}$. We show how some 
previously known or conjectured formulas can be stated using the sweep map together with certain area statistics, and we also give some new formulas and conjectures for rational-slope and rectangular cases.

This paper is an extended abstract of the paper [3]. That full paper contains both proofs as well as additional details on such tasks as how to explicitly invert certain sweep maps.

\section{Partitions, Words, and Lattice Paths}

The fundamental objects considered in this paper live on the integer square lattice within the first quadrant. These objects include integer partitions (as Ferrers diagrams) and lattice paths. Integer parameters $a$ and $b$ will serve to restrict our attention to such objects fitting within an $a \times b$ rectangle. Integer parameters $r$ and $s$ will be used to assign a "level" to the components of the various objects considered. Of particular interest will the case of $r=b$ and $s=-a$. The constraint of $\operatorname{gcd}(a, b)=1$ arises naturally in some particular sweep maps such as the map due to Armstrong, Hanusa and Jones [2] and the map due to Gorksy and Mazin [10, 11] (see Sections 4.2] and 4.3, respectively).

Let $a, b \in \mathbb{Z}_{\geq 0}$. Integer partitions with at most $a$ parts and largest part at most $b$ fit naturally (adhering to the English convention) in the rectangle with vertices $(0,0),(b, 0),(0, a)$ and $(b, a)$. We denote the set of such partitions (as subsets of boxes in the first quadrant) by $\mathcal{R}^{\text {ptn }}=\mathcal{R}^{\operatorname{ptn}}(a, b)$. Let $\{N, E\}^{*}$ denote the set of all words $w=w_{1} w_{2} \cdots w_{n}, n \geq 0$, for which each $w_{j} \in\{N, E\}$ and let $\mathcal{R}^{\text {word }}=$ $\mathcal{R}^{\text {word }}\left(N^{a} E^{b}\right)$ denote the subset of words consisting of $a$ copies of $N$ and $b$ copies of $E$. Finally, let $\mathcal{R}^{\text {path }}=\mathcal{R}^{\text {path }}\left(N^{a} E^{b}\right)$ denote the set of lattice paths from $(0,0)$ to $(b, a)$ consisting of $a$ unit-length "north" steps and $b$ unit-length "east" steps.

There are natural bijections among the three sets $\mathcal{R}^{\text {ptn }}, \mathcal{R}^{\text {word }}$ and $\mathcal{R}^{\text {path }}$. Each word $w \in \mathcal{R}^{\text {word }}$ encodes a lattice path in $\mathcal{R}^{\text {path }}$ by letting each $E$ correspond to an east step and each $N$ correspond to a north step. The "frontier" of a partition $\pi \in \mathcal{R}^{\text {ptn }}$ also naturally encodes a path in $\mathcal{R}^{\text {path }}$. We write $\operatorname{WORD}(P)$ or $\operatorname{WORD}(\pi)$ for the word associated to a path $P$ or a partition $\pi$, respectively. Operators PATH and PTN are defined analogously.

Let $r, s \in \mathbb{Z}$. We assign a level to each square of a partition and each step of a path in the following manner. First, assign to each lattice point $(x, y)$ the $(\boldsymbol{r}, s)$-level $r y+s x$. Assign to each lattice square the level of its southeast corner. We will have occasion to consider two different conventions for associating levels to north and east steps of paths. For the east-north convention, each east (north) step inherits the level of its eastern (northern) endpoint. For the west-south convention, each east (north) step inherits the level of its western (southern) endpoint. Figure 2 illustrates the various levels relevant to the path $N N E N E$ for $r=8$ and $s=-5$.

The set of partitions in $\mathcal{R}^{\mathrm{ptn}}(a, b)$ whose lattice squares have nonnegative $(r, s)$-levels will be denoted $\mathcal{D}^{\text {ptn }}=\mathcal{D}_{r, s}^{\text {ptn }}(a, b)$ and its members will be referred to as $(r, s)$-Dyck partitions. A lattice path $P$ (and its corresponding word, $\operatorname{WORD}(P)$ ) will be termed $(\boldsymbol{r}, \boldsymbol{s})$-Dyck if the underlying partition $\operatorname{PTN}(P)$ is $(r, s)$-Dyck. We use $\mathcal{D}_{r, s}^{\text {path }}\left(N^{a} E^{b}\right)$ and $\mathcal{D}_{r, s}^{\text {word }}\left(N^{a} E^{b}\right)$ to denote the set of such $(r, s)$-Dyck paths and $(r, s)$-Dyck words, respectively. Note that whether or not a path or word is $(r, s)$-Dyck is independent of which level-assignment convention is used.

\section{The Sweep Map}

We begin in Section 3.1 by giving an algorithmic description of the basic sweep maps for two-dimensional lattice paths. We then present a general sweep map in Section 3.2 and various minor variations of the basic sweep map in Section 3.3 . 

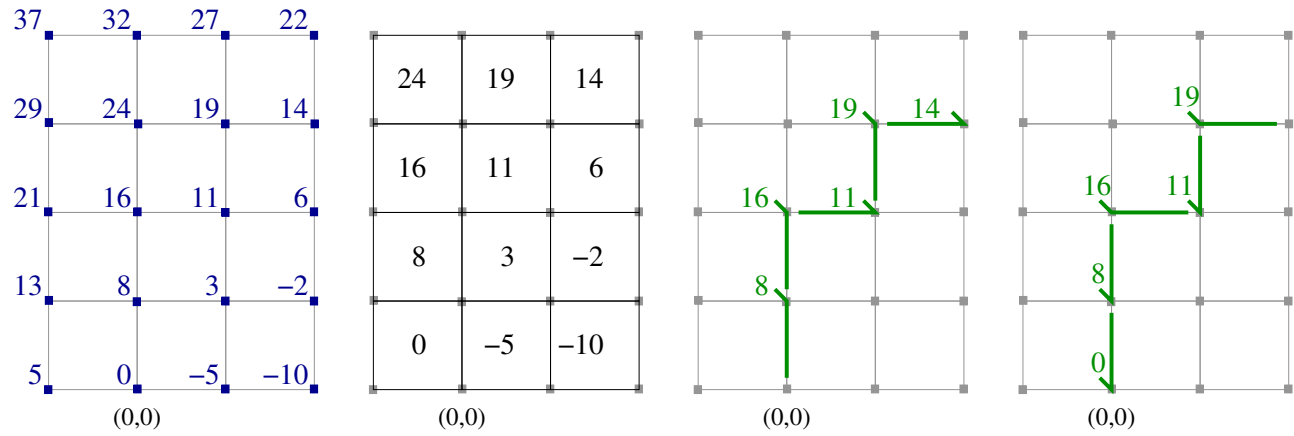

Fig. 2: Illustration of level-assignment conventions for lattice points, squares, path steps with the east-north convention, and path steps with the west-south convention for the case of $r=8$ and $s=-5$.

\subsection{The basic sweep map}

Let $r, s \in \mathbb{Z}$. We first describe the $(\boldsymbol{r}, s)^{-}$-sweep map, $\operatorname{sw}_{r, s}^{-}:\{N, E\}^{*} \rightarrow\{N, E\}^{*}$. Given $w \in$ $\{N, E\}^{*}$, assign levels using the east-north convention applied to PATH $(w)$. Define a word $y=\operatorname{sw}_{r, s}^{-}(w)$ by the following algorithm. Initially, $y$ is the empty word. For $k=-1,-2,-3, \ldots$ and then for $k=$ $\ldots, 3,2,1,0$, scan $w$ from right to left. Whenever a letter $w_{i}$ is encountered with level $k$, append $w_{i}$ to $y$. The $(r, s)^{+}$-sweep map $\mathrm{sw}_{r, s}^{+}$is defined the same way as $\mathrm{sw}_{r, s}^{-}$except that 1$)$ the value 0 is the first value of $k$ used rather than the last and 2) for each value of $k, w$ is scanned from left to right. Figure 3 illustrates the action of both $\mathrm{sw}_{3,-2}^{+}$and $\mathrm{sw}_{3,-2}^{-}$on a path in $\mathcal{R}^{\text {path }}\left(N^{8} E^{10}\right)$. When the distinction between, or choice of, $\mathrm{sw}_{r, s}^{+}$versus $\mathrm{sw}_{r, s}^{-}$is unimportant, we will simply write $\mathrm{sw}_{r, s}$. We define the action of $\mathrm{sw}_{r, s}$ on a partition $\pi$ as $\operatorname{PTN}\left(\operatorname{sw}_{r, s}(\operatorname{WORD}(\pi))\right)$ and the action of $\mathrm{sw}_{r, s}$ on a path as $\operatorname{PATH}\left(\mathrm{sw}_{r, s}(\operatorname{WORD}(P))\right)$.

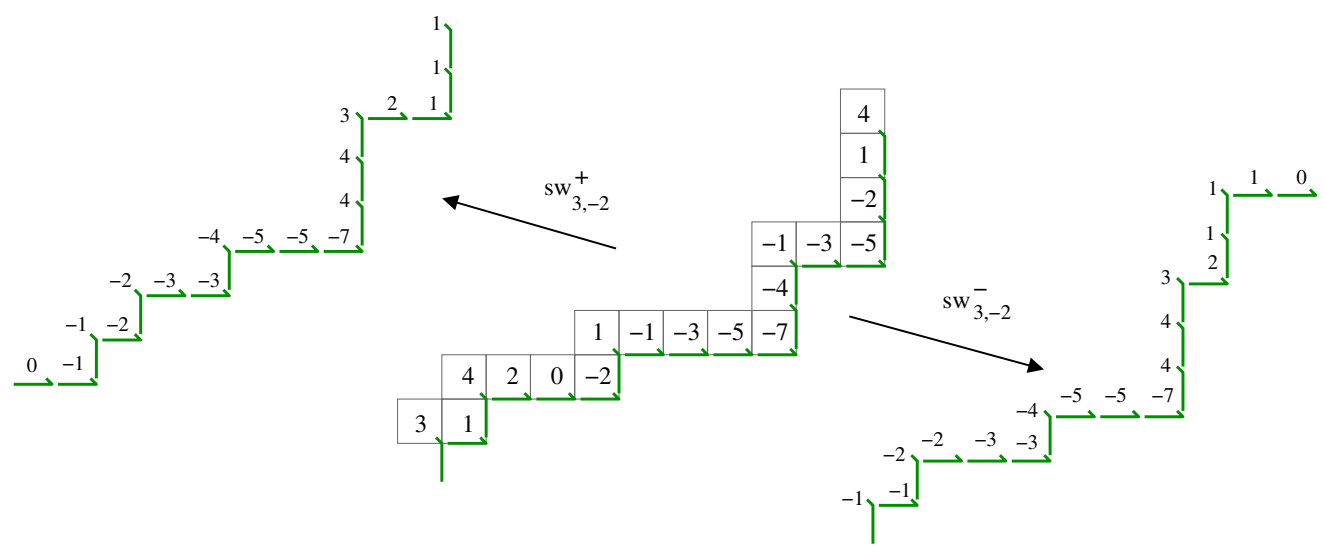

Fig. 3: Images of $\mathrm{sw}_{3,-2}^{-}$and $\mathrm{sw}_{3,-2}^{+}$acting on a path in $\mathcal{R}^{\text {path }}\left(N^{8} E^{10}\right)$.

Geometrically, we think of each step in $\operatorname{PATH}(w)$ as a wand whose tip is located at the lattice point at the end of each step. (The maps $\mathrm{sw}_{r, s}^{ \pm}$defined above are assigning labels according to the east-north convention. Some of the variants described in Section 3.3 assign levels according to the west-south 
convention.) The sweep map $\mathrm{sw}_{r, s}$ acts (at least when $r>0$ and $s<0$ ) by scanning southwest along each diagonal line $r y+s x=c$ in the appropriate order and "sweeping up" the wands whose tips lie on each of these diagonals. The wands are laid out in the order in which they were swept up to produce the output lattice path. (The labels on the wand tips are not part of the final output.) It is clear from this description, or from the original definition, that the sweep map depends only on the "slope" $-s / r$; more precisely, we have $\mathrm{sw}_{r, s}=\mathrm{sw}_{r k, s k}$ for every positive integer $k$.

Remark 3.1 The area* statistic introduced in Section 5.1 uses the actual $(r, s)$-wand labels, and so it's not universally safe to always assume $\operatorname{gcd}(r, s)=1$.

Since the sweep maps merely rearrange the letters in the input word $w$, it is immediate that both $\mathrm{sw}_{r, s}^{-}$ and $\mathrm{sw}_{r, s}^{+}$map each set $\mathcal{R}^{\text {word }}\left(N^{a} E^{b}\right)$ into itself. As described in [3], one can easily extend the basic sweep map to irrational slopes. The irrational-slope sweep maps provides a direct proof of the following.

Proposition 3.2 For all $r, s \in \mathbb{Z}$ and $a, b \geq 0, \mathrm{sw}_{r, s}$ maps each set $\mathcal{D}_{r, s}^{\text {word }}\left(N^{a} E^{b}\right)$ into itself.

Note that we do not have a proof of injectivity for these irrational-slope sweep maps.

\subsection{The general sweep map}

Suppose $A=\left\{x_{1}, \ldots, x_{k}\right\}$ is a given alphabet and wt $: A \rightarrow \mathbb{Z}$ is a function assigning an integer weight to each letter in $A$. Given a word $w=w_{1} w_{2} \cdots w_{n} \in A^{*}$, define the wand labels $l_{0}, \ldots, l_{n}$ relative to the weight function wt by setting $l_{0}=0$ and, for $1 \leq i \leq n$, letting $l_{i}=l_{i-1}+\operatorname{wt}\left(w_{i}\right)$. (These wand labels are essentially computed according to the east-north convention, though the west-south convention works equally well.) Define $\mathrm{sw}_{\mathrm{wt}}: A^{*} \rightarrow A^{*}$ as follows: For each $k$ from -1 down to $-\infty$ and then from $\infty$ down to 0 , scan $w$ from right to left, writing down each $w_{i}$ with $l_{i}=k$ and $i>0$. Let $\mathcal{R}^{\text {word }}\left(x_{1}^{n_{1}} \cdots x_{k}^{n_{k}}\right)$ be the set of words $w \in A^{*}$ consisting of $n_{j}$ copies of $j$ for $1 \leq j \leq k$. Let $\mathcal{D}_{\mathrm{wt}}^{\mathrm{word}}\left(x_{1}^{n_{1}} \cdots x_{k}^{n_{k}}\right)$ be the set of such words for which all labels $l_{i}$ are nonnegative.

Conjecture 3.3 Let $A=\left\{x_{1}, \ldots, x_{k}\right\}$ be an alphabet and wt : $A \rightarrow \mathbb{Z}$ a weight function. For any nonnegative integers $n_{1}, n_{2}, \ldots, n_{k}$,

- $\mathrm{sw}_{\mathrm{wt}}$ maps $\mathcal{R}^{\mathrm{word}}\left(x_{1}^{n_{1}} \cdots x_{k}^{n_{k}}\right)$ bijectively to itself, and

- $\mathrm{sw}_{\mathrm{wt}}$ maps $\mathcal{D}_{\mathrm{wt}}^{\mathrm{word}}\left(x_{1}^{n_{1}} \cdots x_{k}^{n_{k}}\right)$ bijectively to itself.

Remark 3.4 The order in which the levels are traversed is a key ingredient to invertibility. For example, in the case of $a=b=2$ and $r=1, s=-1$, if we scan levels in the order $k=\ldots, 2,1,0,-1,-2, \ldots$, both of the paths NENE and NEEN map to NNEE.

\subsection{Reversed and transposed sweeps}

For fixed $r$ and $s$, there are four natural parameters that can be used to define a potential sweep map:

- The level to start sweeping at,

- The direction of sweep for a given level (i.e., right-to-left or left-to-right),

- The relative order in which to visit levels (i.e., $k+1$ after level $k$ versus $k-1$ after level $k$ ),

- The convention for labeling steps (i.e., assign levels according to the west-south convention or the east-north convention). 
Empirical evidence suggests that for each of the $8=2^{3}$ possible choices for the second through fourth parameters, there is a unique choice of starting level that will lead to an injective sweep map for general $\mathcal{R}^{\text {word }}\left(N^{a} E^{b}\right)$. In fact, each of these maps is closely related to each other through two natural involutions on paths. Let rev : $\{N, E\}^{*} \rightarrow\{N, E\}^{*}$ be the reversal map given by rev $\left(w_{1} w_{2} \cdots w_{n}\right)=w_{n} \cdots w_{2} w_{1}$. Let flip : $\{N, E\}^{*} \rightarrow\{N, E\}^{*}$ be the transposition map that acts on a word $w$ by interchanging $N \mathrm{~s}$ and $E$ s. Evidently, both rev and flip are involutions; rev maps $\mathcal{R}^{\text {word }}\left(N^{a} E^{b}\right)$ bijectively onto itself, whereas flip maps $\mathcal{R}^{\text {word }}\left(N^{a} E^{b}\right)$ bijectively onto $\mathcal{R}^{\text {word }}\left(N^{b} E^{a}\right)$. We can modify the sweep maps by composing on the left or right with rev and/or flip. The new maps are one-to-one (hence bijections between appropriate domains and codomains) iff the original sweep maps are one-to-one. One can check that revo $\mathrm{sw}_{r,-s}^{+}=$flip $\circ \mathrm{sw}_{s,-r}^{-} \circ$ flip. The paper [3] describes explicitly the eight maps along with their relationships to $\mathrm{sw}_{r, s}^{-}$and $\mathrm{sw}_{r, s}^{+}$.

\section{Algorithms Equivalent to a Sweep Map}

This section reviews some algorithms that have appeared in the literature that are equivalent to special cases of variants of the sweep map. We describe each algorithm and indicate its exact relation to the general sweep map. Proofs that each algorithm really does implement a particular case of the sweep map may be found in [3].

\subsection{Algorithms based on area vectors}

\subsubsection{Trapezoidal lattice paths}

In [16], the first author considers analogues of the $q, t$-Catalan numbers associated to paths contained in a trapezoidal region. Fix integers $k \geq 0$ and $n, m>0$. Let $T_{n, k, m}$ denote the trapezoidal lattice paths from $(0,0)$ to $(k+m n, n)$ that never go strictly to the right of the line $x=k+m y$. Loehr introduces two different families of statistics on these paths: one based on bounce paths and the other on generalized inversion statistics. He then introduces a bijection $\phi=\phi_{n, k, m}$ on $T_{n, k, m}$ that proves that the associated generating functions are equal. Below we recall his definition and then show that a variant of $\phi$ is a sweep map.

Given a path $P \in T_{n, k, m}$, we first construct the (slope- $\boldsymbol{m}$ ) area vector $g(P)=\left(g_{0}, g_{1}, \ldots, g_{n-1}\right)$, where $g_{i}$ is the number of complete lattice squares in the horizontal strip $\{(x, y): x \geq 0, i \leq y \leq i+1\}$ that lie to the right of $P$ and to the left of the line $x=k+m y$. The area vector $g(P)$ has the following properties: $0 \leq g_{0} \leq k ; g_{i}$ is a nonnegative integer for $0 \leq i<n$; and $g_{i} \leq g_{i-1}+m$ for $1 \leq i<n$. One readily checks that $P \mapsto g(P)$ is a bijection from $T_{n, k, m}$ to the set of vectors of length $n$ with the properties just stated.

We define the new path $\operatorname{PATH}(v)=\phi(P) \in T_{n, k, m}$ by concatenating lattice paths (regarded as words in $\left.\{N, E\}^{*}\right)$ that are built up from subwords of $g(P)$ as follows. For $i=0,1,2, \ldots, M$, let $z^{(i)}$ be the subword of $g(P)$ consisting of symbols in the set $\{i, i-1, i-2, \ldots, i-m\}$; here $M$ is maximal such that $z^{(i)}$ is nonempty. Create a word $v^{(i)} \in\{N, E\}^{*}$ from $z^{(i)}$ by replacing each symbol $i$ in $z^{(i)}$ by $N$ and replacing all other symbols in $z^{(i)}$ by $E$. Finally, let $v$ be the concatenation of words

$$
v=v^{(0)} E v^{(1)} E v^{(2)} \ldots E v^{(k)} v^{(k+1)} \ldots v^{(M)},
$$

in which an extra east step is added after the first $k$ words. It is proved in [16] that $\operatorname{PATH}(v)=\phi(P)$ always lies in $T_{n, k, m}$, and that $\phi_{n, k, m}$ is a bijection. 
To relate $\phi$ to the sweep map, we need to introduce a modified map $\phi^{\prime}$ that incorporates the bijection described in [16, Sec. 4]. To describe $\phi^{\prime}$, keep the notation of the previous paragraph. For all $i$ with $k<i \leq M$, note that $v^{(i)}$ must begin with an $E$, so we can write $v^{(i)}=E \tilde{v}^{(i)}$. Let $w^{(i)}=\operatorname{rev}\left(v^{(i)}\right)$ for $0 \leq i \leq k$, and let $w^{(i)}=E \operatorname{rev}\left(\tilde{v}^{(i)}\right)$ for $k<i \leq M$. Define $\phi^{\prime}(P)=\operatorname{PATH}(w)$, where

$$
w=w^{(0)} E w^{(1)} E w^{(2)} \ldots E w^{(k)} w^{(k+1)} \ldots w^{(M)} .
$$

Example 4.1 Let $n=8, k=2, m=2$, and $\operatorname{wORD}(P)=$ ENNEENEEEEENNEEENNEEENEEEE. Then $g(P)=(1,3,3,0,2,1,3,2)$, so

$$
\begin{aligned}
& z^{(0)}=0, \quad z^{(1)}=101, \quad z^{(2)}=10212, \quad z^{(3)}=1332132, \quad z^{(4)}=33232, \quad z^{(5)}=333, \\
& v^{(0)}=N, \quad v^{(1)}=N E N, \quad v^{(2)}=E E N E N, \quad v^{(3)}=E N N E E N E, \quad v^{(4)}=\text { EEEEE }, \quad v^{(5)}=E E E, \\
& w^{(0)}=N, \quad w^{(1)}=N E N, \quad w^{(2)}=N E N E E, \quad w^{(3)}=E E N E E N N, \quad w^{(4)}=E E E E E, \quad w^{(5)}=E E E, \\
& \operatorname{wORD}(\phi(P))=N \text { ENEN EEENEN ENNEENE EEEEE EEE, } \\
& \operatorname{WORD}\left(\phi^{\prime}(P)\right)=\text { NENEN ENENEE EENEENN EEEEE EEE. }
\end{aligned}
$$

Theorem 4.2 Suppose $k \geq 0, n, m>0, w \in\{N, E\}^{*}$, and $P=\operatorname{PATH}(w) \in T_{n, k, m}$. Then

$$
\operatorname{WORD}\left(\phi_{n, k, m}^{\prime}(P)\right)=\text { flip } \circ \text { rev } \circ \mathrm{sw}_{1,-m} \circ \operatorname{rev} \circ \mathrm{flip}(w) \text {. }
$$

\subsubsection{Square lattice paths}

In [18], the authors modified the map $\phi_{n, 0,1}$ to obtain a bijection $\phi$ on $\mathcal{R}^{\text {path }}\left(N^{n} E^{n}\right)$. Given $P \in$ $\mathcal{R}^{\text {path }}\left(N^{n} E^{n}\right)$, we define its area vector $g(P)=\left(g_{0}, g_{1}, \ldots, g_{n-1}\right)$ by letting $g_{i}+n-i$ be the number of complete boxes in the strip $\{(x, y): x \geq 0, i \leq y \leq i+1\}$ that lie to the right of $P$ and to the left of $x=n$. (This reduces to the previous slope-one area vector if $P$ is a Dyck path.) In this case, the area vectors of paths in $\mathcal{R}^{\text {path }}\left(N^{n} E^{n}\right)$ are characterized by the following properties: $g_{0} \leq 0 ; g_{i}+n-i \geq 0$ for $0 \leq i<n$; and $g_{i} \leq g_{i-1}+1$ for $1 \leq i<n$.

Given $P \in \mathcal{R}^{\text {path }}\left(N^{n} E^{n}\right)$, we define a new path PATH $(v)=\phi(P)$ as follows. For all $i \in \mathbb{Z}$, let $z^{(i)}$ be the subword of $g(P)$ consisting of all occurrences of $i$ and $i-1$. For all $i \geq 0$, let $y^{(i)}$ be the reversal of $z^{(i)}$. For all $i<0$, let $y^{(i)}$ be the word obtained by reversing $z^{(i)}$, then moving the leftmost symbol (which must be $i$ ) to the right end of the word. Create words $v^{(i)}$ by replacing each $i-1$ in $y^{(i)}$ by $N$ and each $i$ in $y^{(i)}$ by $E$. Finally, define $\operatorname{PATH}(v)$ to be the concatenation of the partial paths $v^{(-1)}, v^{(-2)}, \ldots$ followed by the partial paths $\ldots, v^{(2)}, v^{(1)}, v^{(0)}$.

Example 4.3 Let $P \in \mathcal{R}^{\text {path }}\left(N^{16} E^{16}\right)$ be such that

$$
\operatorname{PATH}(P)=\text { ENEENENNNNEENEEEENNNENEENNNNENEE. }
$$

Then $g(P)=(-1,-2,-2,-1,0,1,0,-3,-2,-1,-1,-2,-1,0,1,1)$, so (for instance) $z^{(1)}=010011$, $y^{(1)}=110010, z^{(-2)}=-2-2-3-2-2, y^{(-2)}=-2-3-2-2-2$, and so on. The new path $\operatorname{PATH}(v)=\phi(P)$ is the concatenation of the word $v^{(-1)}=$ NEENENNEE, $v^{(-2)}=$ ENEEE, $v^{(-3)}=E$, $v^{(2)}=N N N, v^{(1)}=E E N N E N$, and $v^{(0)}=E N N N E E N N$. On the other hand, the reader can check that $\operatorname{PATH}(v)=\operatorname{sw}_{1,-1}(P)$. In fact, for $-3 \leq i \leq 2$, the subword $v^{(i)}$ consists precisely of the steps in the output of the sweep map that came from steps with $(1,-1)$-label equal to $i$ in the input word $\operatorname{WORD}(P)$.

Theorem 4.4 For all $n>0$ and all $P \in \mathcal{R}^{\mathrm{path}}\left(N^{n} E^{n}\right), \phi(P)=\mathrm{sw}_{1,-1}(P)$. 


\subsection{3 q,t-Schröder numbers}

An order- $n$ Schröder path is a path from the origin $(0,0)$ to $(n, n)$ never going below $y=x$ comprised not only of the unit north and east steps, but also of a length- $\sqrt{2}$ northeast step. In [8], the authors define area, dinv and bounce statistics in analogy with their Catalan brethren. In [8, Theorem 6], they define a map $\phi$ that acts on the Schröder paths and prove it sends (dinv, area) to (area, bounce). A direct translation of notation establishes the following:

Theorem 4.5 The map $\phi$ described in [8] Theorem 6] is equivalent to the sweep map $\mathrm{sw}_{\mathrm{wt}}$ associated to the alphabet $A=\{N, D, E\}$ with weight function $\operatorname{wt}(N)=1, \operatorname{wt}(D)=0, \operatorname{wt}(E)=-1$.

\subsection{An algorithm based on hook-lengths}

In [2], D. Armstrong, C. Hanusa and B. Jones investigate the combinatorics of $(a, b)$-cores. In the process, they define a map zeta $: \mathcal{D}_{b,-a}^{\mathrm{ptn}}(a, b) \rightarrow \mathcal{D}_{b,-a}^{\mathrm{ptn}}(a, b)$. Let $\operatorname{gcd}(a, b)=1$ and $\pi \in \mathcal{D}_{b,-a}^{\mathrm{ptn}}(a, b)$. The partition zeta $(\pi)$ is defined in two stages. First, create a partition $\nu=n u(\pi)$ as follows. Consider the labels of all lattice squares lying above $b y=a x$ and below the path PATH $(\pi)$. Sort these labels into increasing order. (It follows from our hypothesis of $\operatorname{gcd}(a, b)=1$ that these labels will all be distinct.) Write them in a column from bottom to top. We define $\nu=\mathrm{nu}(\pi)$ to be the unique partition such that these labels are the hook lengths of the cells in the first column.

The second stage maps $\nu$ to a new partition $\rho=r h \circ(\nu)$ as follows. There will be one row of $\rho$ for each row of $\nu$ whose first-column hook length labels a north step of $\operatorname{PATH}(\pi)$. To determine the length of each row in $\rho$, count the number of cells of $\nu$ in the corresponding row whose hook-length is less than or equal to $b$. The zeta map is then defined by zeta $(\pi)=$ rhoo nu $(\pi)$.

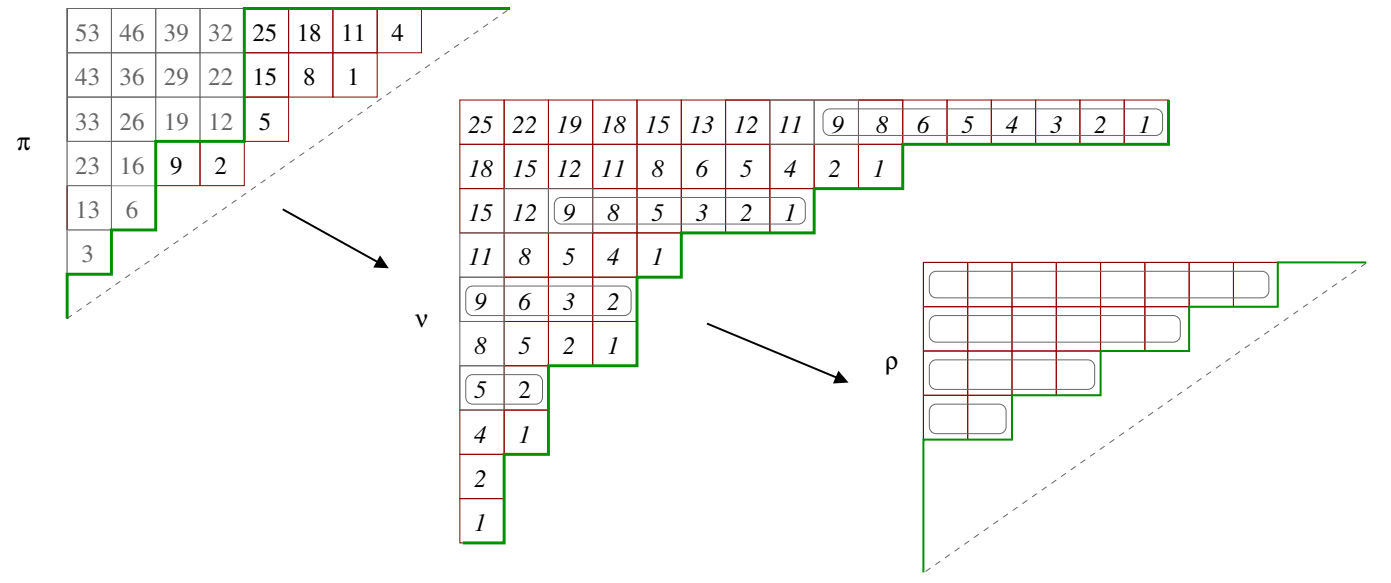

Fig. 4: Example of the zeta map for $\pi=(4,4,4,2,2,1)$ for $a=7$ and $b=10$. The resulting partition $\rho$ is $(8,6,4,2)$ as shown. Note that the numbers written within the squares of $\nu$ are hook lengths rather than the lattice-square labels defined in the Introduction.

Theorem 4.6 For $a, b \in \mathbb{Z}_{>0}$ with $\operatorname{gcd}(a, b)=1$, $\operatorname{WORD}(\operatorname{zeta}(\pi))=\operatorname{sw}_{b,-a}^{+} \circ \operatorname{rev}(\operatorname{WORD}(\pi))$ for all $\pi \in \mathcal{D}_{b,-a}^{\mathrm{ptn}}(a, b)$. 


\subsection{An algorithm based on semi-module generators}

In [10, 11], E. Gorsky and M. Mazin relate the $q, t$-Catalan numbers and their generalizations to the homology of compactified Jacobians for singular plane curves with Puiseux pair $(a, b)$. In the course of their investigations, they introduce a map $G$ on partitions in $\mathcal{D}_{b,-a}^{\mathrm{ptn}}(a, b)$. Theorem 4.8 states that the map $G$ is a sweep map.

Let $a, b \in \mathbb{Z}$ with $\operatorname{gcd}(a, b)=1$ and $\pi \in \mathcal{D}_{b,-a}^{\text {ptn }}(a, b)$. For $1 \leq i \leq b$, define the $\boldsymbol{b}$-generators of $\boldsymbol{\pi}$, $\left\{\beta_{1}<\cdots<\beta_{b}\right\}$, to be the levels of the squares immediately above $\operatorname{PATH}(\pi)$. Define $\Delta=\Delta(\pi)$ to be the set of labels of all lattice squares lying above $\operatorname{PATH}(\pi)$ (i.e., including squares not adjacent to $\operatorname{PATH}(\pi)$ ). We then define a new partition $\rho=G(\pi)$ by setting the $i$-th column of $\rho$ to have length

$$
g_{b}\left(\beta_{i}\right)=\left|\left\{\left\{\beta_{i}, \beta_{i}+1, \ldots, \beta_{i}+a-1\right\} \backslash \Delta\right\}\right| .
$$

Example 4.7 Consider $\pi=(4,4,4,2,2,1), a=7$ and $b=10$ as in Section 4.2 The 10-generators in this case are $\{0,3,6,7,12,14,19,21,28,35\}$ and

$$
\Delta=\mathbb{Z}_{\geq 0} \backslash\{1,2,4,5,8,9,11,15,18,25\} .
$$

It follows that $\left(g_{10}(0), g_{10}(3), \ldots, g_{10}(35)\right)=(4,4,3,3,2,2,1,1)$. These are the column lengths of the partition $(8,6,4,2)$, as desired.

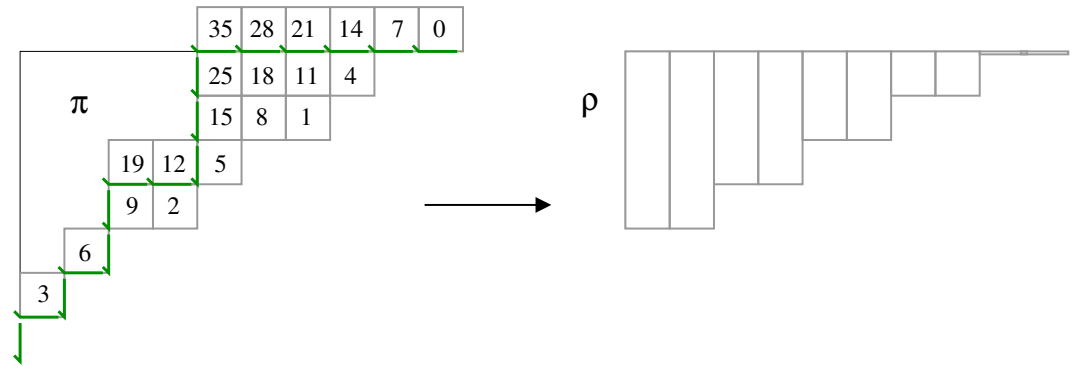

Fig. 5: Example of the Gorsky-Mazin map.

Theorem 4.8 For $a, b \in \mathbb{Z}_{>0}$ with $\operatorname{gcd}(a, b)=1, \operatorname{WORD}(G(\pi))=\mathrm{sw}_{b,-a}^{+} \circ \operatorname{rev}(\operatorname{WORD}(\pi))$ for all $\pi \in \mathcal{D}_{b,-a}^{\mathrm{ptn}}(a, b)$.

\section{Area Statistics and Generalized $q, t$-Catalan Numbers}

This section applies the sweep map to provide new combinatorial generalizations of the $q, t$-Catalan numbers [9] and the $q, t$-square numbers [18]. Several of the generating functions introduced below have (at least conjecturally) close relations to the nabla operator $\nabla$ on symmetric functions introduced by A. Garsia and F. Bergeron [4, 5, 6]. See [3] for details.

\subsection{Area statistics}

For any word $w \in\{N, E\}^{*}$, let area $(w)$ be the number of pairs $i<j$ with $w_{i}=E$ and $w_{j}=N$. This is the area of the partition diagram consisting of the boxes above and to the left of the steps in the lattice 
path encoded by $w$. For $r, s \in \mathbb{Z}$, let $w$ have $(r, s)$-labels $l_{0}, l_{1}, \ldots$ Let $\mathrm{ml}_{r, s}(w)=\min \left\{l_{0}, l_{1}, \ldots\right\}$, and set $\operatorname{area}_{r, s}^{*}(w)=\operatorname{area}(w)+\mathrm{ml}_{r, s}(w)$. Note that $\operatorname{area}_{r, s}^{*}(w) \neq \operatorname{area}_{r k, s k}^{*}(w)$ in general, so we cannot necessarily assume that $\operatorname{gcd}(r, s)=1$ when using area*.

\subsection{Generalized $q, t$-Catalan polynomials}

For $r, s \in \mathbb{Z}$ and $a, b \geq 0$, define the $q, t$-Catalan numbers for slope $(-s / r)$ ending at $(b, a)$ by

$$
C_{r, s, a, b}(q, t)=\sum_{w \in \mathcal{D}_{r, s}^{\text {word }}\left(N^{a} E^{b}\right)} q^{\operatorname{area}(w)} t^{\operatorname{area}\left(\operatorname{sw}_{r, s}(w)\right)} .
$$

Conjecture 5.1 (Joint Symmetry) For all $r, s \in \mathbb{Z}, a, b \geq 0, C_{r, s, a, b}(q, t)=C_{r, s, a, b}(t, q)$.

Note that the conjectured bijectivity of $\operatorname{sw}_{r, s}$ on the domain $\mathcal{D}_{r, s}^{\text {word }}\left(N^{a} E^{b}\right)$ would imply the weaker univariate symmetry property $C_{r, s, a, b}(q, 1)=C_{r, s, a, b}(1, q)$.

\subsection{Generalized $q, t$-square numbers}

Next we generalize the $q, t$-square numbers studied in [18]. For $a, b \geq 0$, define the $\boldsymbol{q}, \boldsymbol{t}$-rectangle numbers for the $a \times b$ rectangle by

$$
S_{a, b}(q, t)=\sum_{w \in \mathcal{R}^{\text {word }}\left(N^{a} E^{b}\right)} q^{\operatorname{area}_{b,-a}^{*}(w)} t^{\operatorname{area}_{b,-a}^{*}\left(\operatorname{sw}_{b,-a}(w)\right)} .
$$

Conjecture 5.2 (Joint Symmetry) For all $a, b \geq 0, S_{a, b}(q, t)=S_{a, b}(t, q)$.

The joint symmetry conjecture is known to hold when $a=b$. This follows from the stronger statement

$$
(q t)^{n(n-1) / 2} S_{n, n}(1 / q, 1 / t)=2\left\langle(-1)^{n-1} \nabla\left(p_{n}\right), s_{\left(1^{n}\right)}\right\rangle,
$$

which was conjectured in [18] and proved in [7]. We conjecture the following more general relationship between certain $q, t$-rectangle numbers and higher powers of $\nabla$.

Conjecture 5.3 For all $m \geq 0$ and $n \geq 1$,

$$
(q t)^{m\left(\begin{array}{c}
n \\
2
\end{array}\right)} S_{n, m n}(1 / q, 1 / t)=(-1)^{n-1}(m+1)\left\langle\nabla^{m}\left(p_{n}\right), s_{\left(1^{n}\right)}\right\rangle .
$$

\subsection{Specialization at $t=1 / q$}

Recall the definitions of $q$-integers, $q$-factorials, and $q$-binomial coefficients: $[n]_{q}=1+q+q^{2}+\cdots+q^{n-1}$, $[n] !_{q}=[n]_{q}[n-1]_{q} \cdots[2]_{q}[1]_{q}$, and $\left[\begin{array}{c}a+b \\ a, b\end{array}\right]_{q}=[a+b] !_{q} /\left([a] !_{q}[b] !_{q}\right)$.

Conjecture 5.4 For all $m, n \geq 0$,

$$
q^{m\left(\begin{array}{c}
n \\
2
\end{array}\right)} S_{n, m n}(q, 1 / q)=\frac{(m+1)}{[m+1]_{q^{n}}}\left[\begin{array}{c}
m n+n \\
m n, n
\end{array}\right]_{q} .
$$

This conjecture generalizes to arbitrary rectangles as follows:

Conjecture 5.5 For all $a, b \geq 0$, write $b=b^{\prime} k$ and $a=a^{\prime} k$ for integers $a^{\prime}, b^{\prime}, k \geq 0$ with $\operatorname{gcd}\left(a^{\prime}, b^{\prime}\right)=1$. The equality

holds $\left(c_{a^{\prime}, b^{\prime}, k}\right.$ is a constant $)$.

$$
q^{c_{a^{\prime}, b^{\prime}, k}} S_{a, b}(q, 1 / q)=\frac{\left(a^{\prime}+b^{\prime}\right)}{\left[a^{\prime}+b^{\prime}\right]_{q^{k}}}\left[\begin{array}{c}
a+b \\
a, b
\end{array}\right]_{q}
$$




\section{Acknowledgements}

The authors gratefully acknowledge discussions with Drew Armstrong, Jim Haglund, Mark Haiman and Michelle Wachs.

\section{References}

[1] George Andrews, Christian Krattenthaler, Luigi Orsina, and Paolo Papi, "ad-Nilpotent b-ideals in $s l(n)$ having a fixed class of nilpotence: combinatorics and enumeration," Trans. Amer. Math. Soc. 354 no. 10 (2002), 3835-3853.

[2] Drew Armstrong, Christopher R. H. Hanusa, and Brant C. Jones, "Results and conjectures on simultaneous core partitions," http://arxiv.org/abs/1308.0572.

[3] Drew Armstrong, Nicholas A. Loehr, and Gregory S. Warrington, "Sweep maps: A continuous family of sorting algorithms," (tentative title) in preparation.

[4] F. Bergeron and A. Garsia, "Science Fiction and Macdonald Polynomials," CRM Proceedings and Lecture Notes AMS VI 3 (1999), 363-429.

[5] F. Bergeron, N. Bergeron, A. Garsia, M. Haiman, and G. Tesler, "Lattice Diagram Polynomials and Extended Pieri Rules," Adv. in Math. 2 (1999), 244-334.

[6] F. Bergeron, A. Garsia, M. Haiman, and G. Tesler, "Identities and Positivity Conjectures for some remarkable Operators in the Theory of Symmetric Functions," Methods and Applications of Analysis VII 3 (1999), 363-420.

[7] Mahir Can and Nicholas A. Loehr, "A proof of the $q, t$-square conjecture," J. Combin. Theory Ser. A 113 (2006), 1419-1434.

[8] Eric Egge, Jim Haglund, Kendra Killpatrick and Darla Kremer, "A Schröder generalization of Haglund's statistic on Catalan paths," Electron. J. of Combin. 10 (2003), Research Paper 16, 21 pages (electronic).

[9] Adriano Garsia and Mark Haiman, "A remarkable $q, t$-Catalan sequence and $q$-Lagrange inversion," J. Algebraic Combin. 5 (1996), 191-244.

[10] Evgeny Gorsky and Mikhail Mazin, "Compactified Jacobians and $q, t$-Catalan numbers, I," J. Combin. Theory Ser. A 120 (2013), 49-63.

[11] Evgeny Gorsky and Mikhail Mazin, "Compactified Jacobians and $q, t$-Catalan numbers, II," J. Algebraic Combin. 39 (2014), no. 1, 153-186.

[12] James Haglund, "Conjectured statistics for the $q, t$-Catalan numbers," Adv. in Math. 175 (2003), 319-334.

[13] James Haglund and Nicholas A. Loehr, "A conjectured combinatorial formula for the Hilbert series for diagonal harmonics," Discrete Math. 298 (2005), 189-204. 
[14] Nicholas A. Loehr, "Conjectured statistics for the higher $q, t$-Catalan sequences," Electron. J. Combin. 12 (2005) research paper R9; 54 pages (electronic).

[15] Nicholas A. Loehr, Multivariate analogues of Catalan numbers, parking functions, and their extensions. Doctoral dissertation, University of California at San Diego (2003), 267 pages.

[16] Nicholas A. Loehr, "Trapezoidal lattice paths and multivariate analogues," Adv. in Appl. Math. 31 (2003), 597-629.

[17] Nicholas A. Loehr and Gregory S. Warrington, "A continuous family of partition statistics equidistributed with length," J. Combin. Theory Ser. A 116 (2009), 379-403.

[18] Nicholas A. Loehr and Gregory S. Warrington, "Square $q$, t-lattice paths and $\nabla\left(p_{n}\right)$," Trans. Amer. Math. Soc. 359 (2007), 649-669.

[19] J. Vaillé, "Une bijection explicative de plusieurs propriétés remarquables des ponts," European J. Combin. 18 (1997), no. 1, 117-124. 